\title{
Strategies for Treatment in Alexander Disease
}

\author{
Albee Messing, ${ }^{* \dagger}$ Christine M. LaPash Daniels, ${ }^{*}$ and Tracy L. Hagemann* \\ *Waisman Center and ${ }^{\dagger}$ Department of Comparative Biosciences, School of Veterinary Medicine, University of Wisconsin- \\ Madison, Madison, Wisconsin 53705
}

\begin{abstract}
Summary: Alexander disease is a rare and generally fatal disorder of the CNS, originally classified among the leukodystrophies because of the prominent myelin deficits found in young patients. The most common form of this disease affects infants, who often have profound mental retardation and a variety of developmental delays, but later onset forms also occur, sometimes with little or no white matter pathology at all. The pathological hallmark of Alexander disease is the inclusion body, known as Rosenthal fiber, within the cell bodies and processes of astrocytes. Recent genetic studies identified heterozygous missense mutations in glial fibrillary acidic protein (GFAP), the major intermediate filament protein in astrocytes, as the cause of nearly all cases of Alexander disease. These
\end{abstract}

studies have transformed our view of this disorder and opened new directions for investigation and clinical practice, particularly with respect to diagnosis. Mechanisms by which expression of mutant forms of glial fibrillary acidic protein (GFAP) lead to the pleiotropic manifestations of disease (afflicting cell types beyond the ones expressing the mutant gene) are slowly coming into focus. Ideas are beginning to emerge that suggest several compelling therapeutic targets for interventions that might slow or arrest the evolution of the disease. This review will outline the rationale for pursuing these strategies, and highlight some of the critical issues that must be addressed in the planning of future clinical trials. Key Words: GFAP, glial fibrillary acidic protein, $\alpha \mathrm{B}$-crystallin, glutamate transporters, Nrf2.

\section{ALEXANDER DISEASE-OVERVIEW}

Alexander disease $(\mathrm{AxD})$ is now recognized as the first primary disorder of astrocytes, resulting as it does from mutations in the intermediate filament protein, glial fibrillary acidic protein (GFAP). ${ }^{1}$ In the most widely recognized form, children present before the age of 2 years with megalencephaly and white matter loss, especially in the frontal lobes. Seizures and spasticity are prominent symptoms, as are hydrocephalus and psychomotor developmental delay. These children suffer progressive deterioration with death usually before the age of 10 .

The key diagnostic feature of the neuropathology in $\mathrm{AxD}$, highlighted in the first description of the disease, ${ }^{2}$ is widespread deposition of Rosenthal fibers in subpial, periventricular, and white matter astrocytes throughout the CNS. ${ }^{3}$ Morphologically, Rosenthal fibers consist of two components: bundles of intermediate filaments surrounding irregular deposits of dense material (FIG. 1). Biochemically, Rosenthal fibers are composed of a com-

Address correspondence and reprint requests to: Albee Messing, V.M.D., Ph.D., University of Wisconsin-Madison, 1500 Highland Ave, Room 713, Madison, WI 53705. E-mail: messing@waisman.wisc.edu. plex ubiquitinated mixture of GFAP in association with other constituents, especially the small stress proteins $\alpha \mathrm{B}$-crystallin and Hsp27. ${ }^{3-5}$

In 2001, we reported the results from sequencing the GFAP gene in a group of 13 patients who had died of biopsy-proven or autopsy-proven AxD. ${ }^{6}$ Twelve of these patients carried nonconservative, heterozygous point mutations in the coding region of GFAP. After this initial report, a number of other studies have now confirmed and extended these findings (reviewed in Reference 1). A diagram showing the location of nearly all published (as well as some unpublished) mutations in relation to the protein domains of GFAP is shown in FIG. 2. Web sites that continue to provide current updates on GFAP mutations can be found at the University of WisconsinMadison (http://www.waisman.wisc.edu/alexander/) and the Human Intermediate Filament Mutation Database (http://www.interfil.org).

Mutations are present in nearly all $(>90 \%)$ cases of AxD. ${ }^{1}$ Nearly one-third involve either of two amino acids (R79 or R239), although it appears that mutations distributed throughout the protein produce essentially identical Rosenthal fibers and similar disease. ${ }^{7}$ All of the mutations are heterozygous, acting in an autosomal dominant fashion. Nearly all mutations are missense muta- 


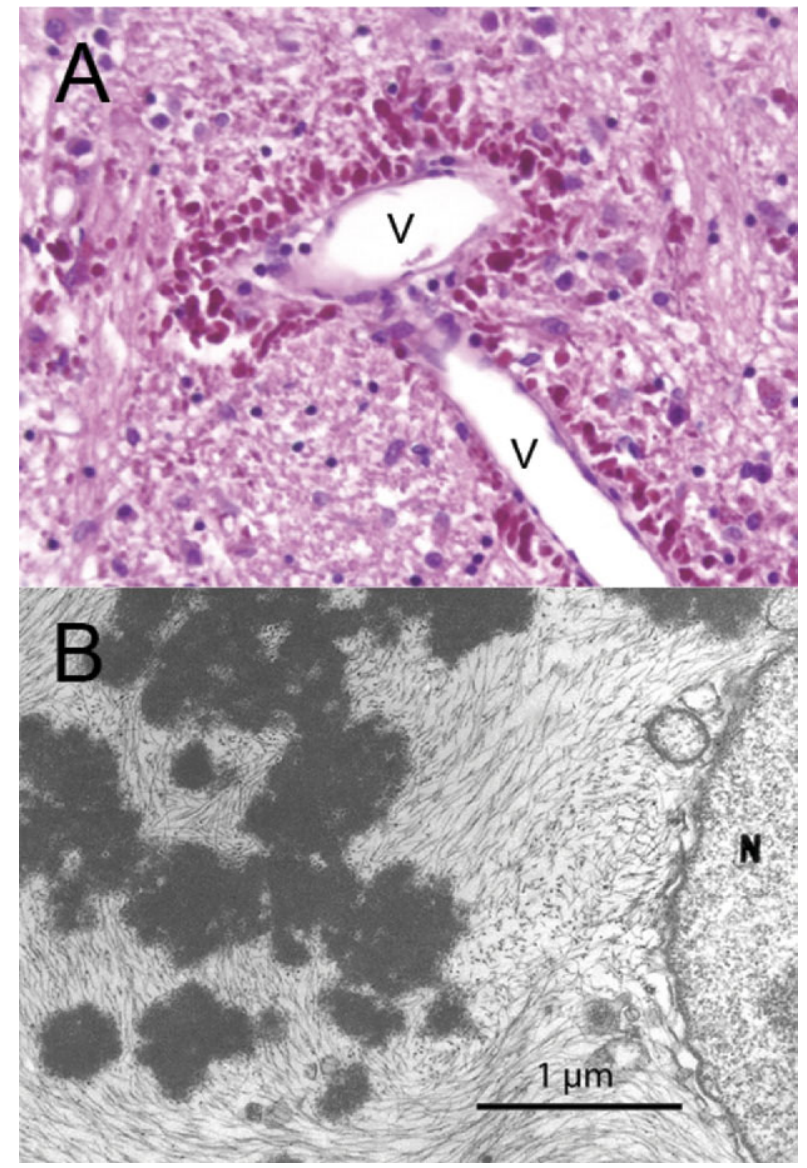

FIG. 1. Rosenthal fibers, shown as eosinophilic aggregates when stained with hematoxylin \& eosin, in astrocyte end-feet surrounding a blood vessel in the brainstem of a child with an R239H mutation (A, reprinted from The Lancet Neurology [2003; 2:75], with permission from Elsevier). Ultrastructural appearance of Rosenthal fibers in an astrocyte cell body (B, reprinted from Eng et al. J Neurosci Res [1998;53:353-60], copyright by WileyLiss, Inc.).

tions, predicting a change in a single amino acid, although recently several other types of mutations have been described with short in-frame insertions or deletions. Proof that mutant protein is actually synthesized, and incorporated as part of the Rosenthal fibers, has been possible for one mutation, R416W. ${ }^{8}$ Most mutations occur de novo, although some adult-onset forms of the disease (where patients live to reproductive age) are transmitted to subsequent generations. The penetrance also approaches $100 \%$, the few exceptions being ones where it can be difficult to distinguish between variable penetrance and presymptomatic diagnosis. ${ }^{1}$

\section{Clinical course and current treatments}

Although AxD is genetically homogeneous, the clinical features of AxD are less consistent. Early descriptions emphasized macrocephaly, frontal leukodystrophy, and a variety of developmental delays; based on reliable genetic diagnoses and larger numbers of patients, it is now clear that these features are not always present. ${ }^{1}$ In particular, later-onset patients often display a very different picture than early-onset patients, dominated by bulbar or pseudo-bulbar signs, atrophy of the cervical spinal cord, and sometimes little or even no leukodystrophy at all..$^{9,10}$ The disorder is typically progressive, with median survivals from the time of onset of 3.6 years for the infantile group, 8 years for the juvenile group, and highly variable durations for the adult group. MRI criteria for diagnosis have been published, ${ }^{9,11}$ although atypical MRIs are also now recognized. ${ }^{12}$ Brenner et al. ${ }^{1}$ have argued that the age distribution of patients is almost certainly skewed by selection bias and that many adultonset patients are missed due to ambiguities in presenting signs, atypical MRIs, and a failure to test for GFAP mutations.

Vanderver has recently proposed a slightly different classification system, focusing less on the age of onset than on constellations of symptoms and regions of MRI lesions that categorize patients as either type I (most of whom would have previously been considered "infantile") or type II (most of whom would have previously been considered juvenile or adult). (A. Vanderver, personal communication).

The current standard of treatment for AxD is symptomatic, focusing on major problems, such as seizure control, nutrition, and maintenance of pulmonary function. Only three reports describe attempts at alternative forms of therapy. One patient who was studied prior to the discovery of GFAP mutations as the cause of the disease was given bone marrow transplantation, which was based on the mistaken analogy of other leukodystrophies that are treated in such a manner. ${ }^{13}$ The patient died 4.5 months after transplantation at the age of 1 year. A second patient (a 9-year-old girl with a D360V mutation) was treated with thyrotropin-releasing hormone (first intravenously, followed by an oral administration) ${ }^{14}$ which was based on the apparent, but still poorly understood, neurotrophic functions of this hormone and its use in improving symptoms in mice and some human patients with other disorders of the hindbrain. One week of intravenous treatment was associated with a reduction in some of her most prominent symptoms, such as vomiting and truncal ataxia, and these improvements persisted through several months after a switch to oral therapy. Other manifestations of the disease were unchanged, such as EEG and MRI abnormalities. Most recently, a 39-year-old woman with an R70Q mutation was treated with a 20-month course of intravenous ceftriaxone, based on the proposed use of this drug for enhancing glutamate transport in astrocytes. ${ }^{15}$ Compared with the 20-month period prior to initiation of treatment, deterioration in gait ataxia, dysarthria, and palatal myoclonus apparently slowed, and the amplitude of evoked nystagmus was reduced. Her ability to read improved, but the MRI was unchanged. Overall, the effectiveness 


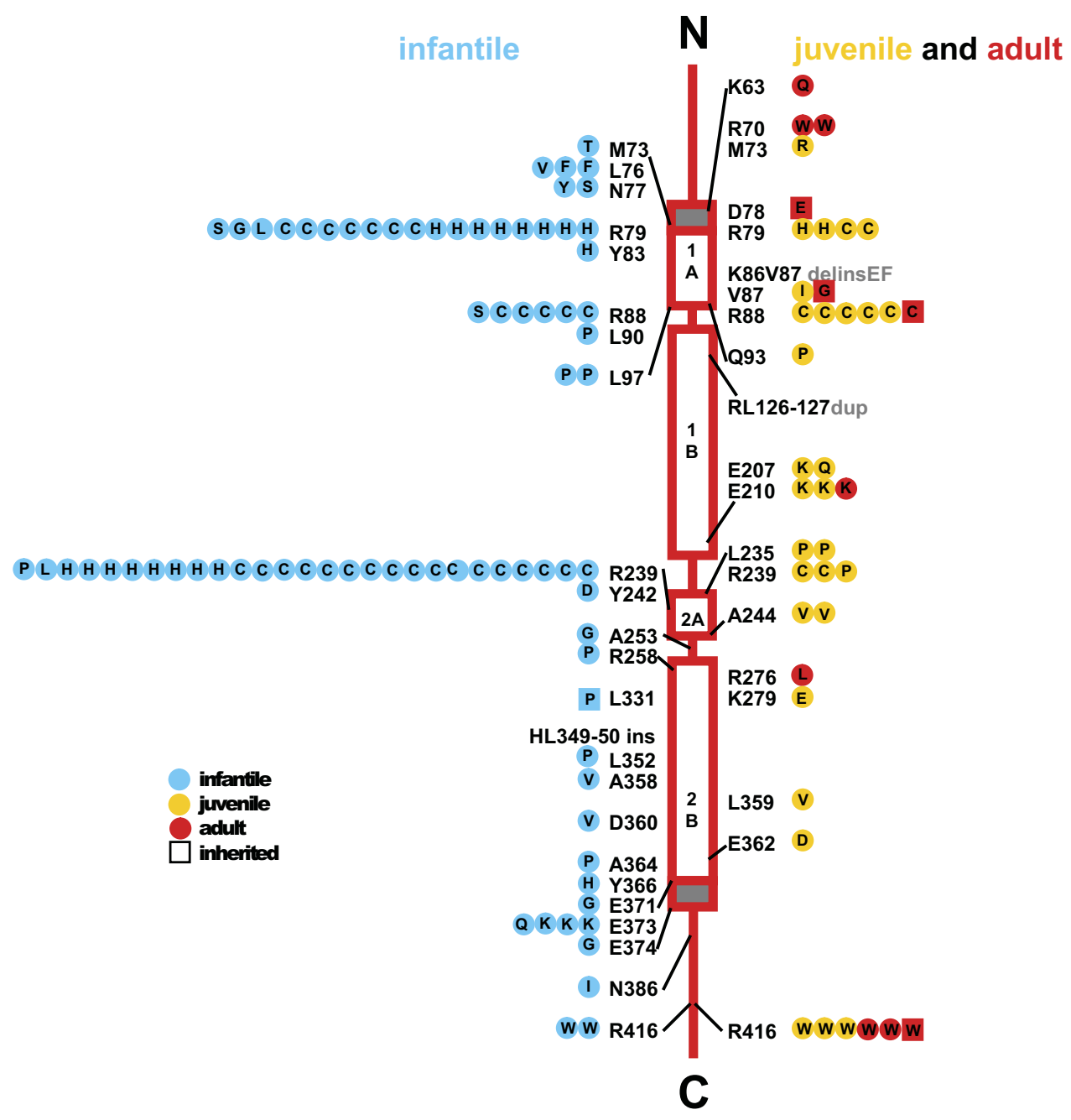

FIG. 2. Locations of published Alexander disease-associated mutations in glial fibrillary acidic protein (GFAP) in relation to protein domain structure of intermediate filaments. The boxes indicate the four $\alpha$-helical subdomains within the central rod domain, separated by nonhelical linkers. The gray box just before segment $1 \mathrm{~A}$ is a nonconserved prehelical sequence important for initiation of rod formation at the start of $1 \mathrm{~A}$; the gray box at the end of 2B represents the highly conserved 365TYRKLLEGEE374 sequence that includes the end of the coiled coil $2 \mathrm{~B}$ segment. Each symbol represents an individual patient, or a family in the case of inherited mutations, or identical twins. Multiple independent occurrences of a mutation are indicated by the number of symbols. Symbols are color coded for clinical category based on age of onset (infantile, juvenile, or adult). The wild type amino acid is indicated next to the structure, and amino acid replacements within symbols on either side. (Adapted and reprinted from Reference 1, with permission from Springer.)

of these latter two treatments is difficult to judge from studies of single patients, especially in the absence of standardized clinical measures and clearly recognized benchmarks for success.

\section{GFAP mutations and gain-of-function hypothesis}

Many of the GFAP mutations associated with AxD occur within amino acid sequences that are highly conserved among intermediate filament proteins. ${ }^{16}$ However, in contrast to most mutations of other intermediate filaments, which lead to loss of function, GFAP mutations appear to produce a toxic gain of function (for review, see Reference 1). The implication of these concepts for devising treatment strategies is that rather than replacing GFAP, we should strive to eliminate the mu- tant protein, prevent its initial expression, or interfere with the consequences of its expression.

As previously noted, the hallmark feature of the pathology, with or without significant leukodystrophy, is the presence of abundant Rosenthal fibers throughout the CNS. What role the Rosenthal fibers play in disease pathogenesis is much less certain. ${ }^{7,17}$ Rosenthal fibers clearly occur in contexts other than $\mathrm{AxD}$, and transgenic mouse studies demonstrate that sufficient over-expression of wild-type human GFAP will produce Rosenthal fibers. ${ }^{18}$ Both knock-in and transgenic studies prove that expression of mutant protein leads to Rosenthal fibers, but again in the context of overexpression. ${ }^{19,20}$ Surprisingly, none of the existing mouse models, while forming 
Rosenthal fibers, have any apparent deficits in white matter, although the ones expressing mutant GFAPs do exhibit abnormal sensitivity to kainic acid-induced seizures, which might model the seizures in the human phenotype. ${ }^{19,20}$ Cell culture studies implicate overexpression and accumulation of GFAP above a currently unknown toxic threshold in the activation of multiple stress pathways and inhibition of proteasome function, with the likelihood of positive feedback loops to cause still further accumulation. ${ }^{21}$ One hypothesis regarding Rosenthal fibers is that the aggregates sequester certain proteins away from critical functions elsewhere in the cell (ie, see below for $\alpha \mathrm{B}$-crystallin). ${ }^{7}$ Another is that the aggregates remove mutant GFAP from the cytoskeleton; thus they have a protective function. The composition of Rosenthal fibers is a topic of active investigation, but at the very least they are now known to contain both wild type and mutant GFAP, stress proteins such as $\alpha \mathrm{B}$-crystallin, Hsp27, intermediate filament-binding partners such as plectin, ${ }^{22}$ and other proteins such as $\mathrm{p} 62 .{ }^{23}$

\section{Mechanisms of leukodystrophy}

Why there is a leukodystrophy in Alexander disease is not currently understood. Depending on the age of onset one can imagine either hypomyelination or demyelination taking place. One possible mechanism for oligodendrocyte or myelin toxicity is exposure to excess levels of glutamate. ${ }^{24-27}$ Astrocyte expression of their major glutamate transporter (Glt-1) is impaired in Alexander disease, ${ }^{28}$ which could pose particular problems for white matter where local release of glutamate occurs from axons, astrocytes, or oligodendrocytes themselves. ${ }^{24,29,30}$ Another possible mechanism for oligodendrocyte toxicity is tumor necrosis factor- $\alpha,{ }^{31}$ the expression of which is elevated in tissue samples from Alexander disease patients and mouse models of the disorder. ${ }^{32}$ It is also conceivable that astrocyte dysfunction in Alexander disease extends to gap junctional communication, and recent studies indicate that astrocyte-targeted double deletion of connexins 43 and 30 results in vacuolation in oligodendrocyte cell bodies and intra-myelin edema. ${ }^{33}$

\section{STRATEGIES FOR THERAPY}

Despite the incomplete state of our knowledge regarding how GFAP mutations cause AxD, a number of useful themes have emerged that suggest potentially effective strategies for therapy. These are presented below in three general groups: 1) reducing the initial insult, 2) enhancing protective stress responses, and 3) minimizing detrimental downstream effects. We are still at an early stage of thinking these problems through, and we offer the following ideas as a guide for future research.

\section{Reducing the initial insult-GFAP}

Expression of mutant GFAP is the root cause of $\mathrm{AxD}$, and accumulation (of both wild type and mutant protein) above a toxic threshold is believed to be an essential element in pathogenesis. Hence, reducing the level or expression of GFAP is one obvious strategy for treatment. An ideal drug might selectively prevent expression of just the mutant allele, but this goal is technically challenging and also involves approaches (such as RNA interference) that have not yet reached clinical practice for any disease. Alternatively, and especially in light of the fact that complete absence of GFAP produces such a mild phenotype in the mouse, ${ }^{34-37}$ one approach being pursued is to screen libraries of drugs or compounds for suppressors of GFAP expression from both alleles. New tools for evaluating regulation of the GFAP promoter in transgenic mice using luciferase reporters have been developed that make such screening feasible. ${ }^{38,39}$ Cho et al. ${ }^{39 a}$ have recently completed a screen using primary cultures of astrocytes derived from normal newborn mice, and they found several well-known drugs that reduce GFAP levels in vitro by $50 \%$ or more. Whether these findings can be easily translated into in vivo treatments, and eventually to humans, remains to be seen.

Instead of reducing initial GFAP synthesis through regulation of the GFAP promoter, an alternative strategy to reduce protein levels is to increase degradation. Although the normal pathways of GFAP degradation are not fully understood, impairment of proteasome function has been proposed as both a cause and a consequence of excess GFAP. ${ }^{21}$ Pharmacological enhancers of proteasome function are therefore a theoretical possibility for treating $\mathrm{AxD}$, but few studies exist on this topic. ${ }^{40}$

In contrast, autophagy appears to be enhanced in $\mathrm{AxD}$, based on data from cell cultures and mouse models, as well as tissues from patients. ${ }^{41}$ One strategy for treatment may be to boost the autophagic response still further, and a number of drugs have already been studied for such properties in the treatment of other neurological disorders. For instance, rapamycin and its analogues can increase autophagy through inhibition of the mammalian target of rapamycin (mTOR), and have recently shown beneficial effects when tested in mouse models of Huntington's disease, ${ }^{42}$ tuberous sclerosis, ${ }^{43,44}$ and spinocerebellar ataxia type $3 .^{45}$ Chronic treatments with rapamycin are tolerated, and even extend lifespan in mice. ${ }^{46}$ Rapamycin also decreased GFAP levels in astrocytoma cell lines expressing AxD mutant GFAP. ${ }^{41}$

A second autophagy-inducing drug is lithium, which reduced cell death and enhanced the clearance of aggregate-prone mutant huntingtin and $\alpha$-synuclein in cell culture models of Huntington's and Parkinson's disease. ${ }^{47,48}$ Lithium also increased autophagy, delayed motoneuron death, increased lifespan, and reduced SOD1 aggregates in a mouse model of amyotrophic lateral scle- 
rosis, ${ }^{49}$ reduced toxicity in a Drosophila model of Huntington's disease, ${ }^{50}$ and reduced degeneration and aggregated tau in a mouse tauopathy model. ${ }^{51}$ Lithium increases autophagy in an mTOR-independent manner through the inhibition of inositol monophosphatase 1, which leads to a depletion of free inositol and myoinositol-1,4,5-triphosphate. ${ }^{48}$ Interestingly, lithium also decreases autophagy by activating mTOR through a pathway involving inhibition of glycogen synthase kinase- $3 \beta .^{52}$ Because lithium has opposing effects on autophagy through the inositol monophosphatase 1 and mTOR pathways, it has been proposed that dual treatment with lithium and rapamycin should cause an additive increase in autophagy through their actions on both the inositol monophosphatase 1 and mTOR pathways. When this strategy was tested in cell culture and Drosophila, lithium and rapamycin together did have an additive effect for increasing autophagy and decreasing mutant protein. ${ }^{48,52}$ Hence combination therapies may ultimately prove necessary for effective treatment of AxD.

\section{Enhancing protective stress responses- $\alpha \mathrm{B}$-crystallin}

Induction of various stress proteins is a prominent feature of protein aggregation disorders, where they are believed to represent an attempt by cells to deal with the challenge of mis-folded or mutant proteins. Hsp70 in particular has been the focus of considerable attention for the treatment of neurodegenerative diseases. Interestingly, Hsp70 is not induced in $\mathrm{AxD}$, and instead the stress response is dominated by small stress proteins such as $\alpha \mathrm{B}$-crystallin and Hsp27. ${ }^{8,53}$ We focus here on $\alpha \mathrm{B}$-crystallin, although many of the same concepts might apply to Hsp27 as well.

$\alpha \mathrm{B}$-crystallin is normally expressed at low levels in the brain, but it is increased in a variety of neurodegenerative disorders ${ }^{54}$ and has been of particular interest for astrocytes and AxD since it was identified as a major component of Rosenthal fibers. ${ }^{4}$ It is also upregulated in the mouse models that develop Rosenthal fibers. ${ }^{18,55}$ In several types of cell culture models of injury or stress, including heat shock (glioma cells) ${ }^{56}$ hypertonicity (kidney, primary astrocytes, glioma cells, heart), ${ }^{57-60}$ and ischemia/hypoxia (heart), ${ }^{60,61}$ the expression of $\alpha \mathrm{B}$-crystallin is markedly upregulated and increases cellular resistance. In some cases $\alpha \mathrm{B}$-crystallin is coordinately upregulated along with $\mathrm{Hsp} 27$, as with cadmium exposure in astrocytes, ${ }^{58}$ but in other situations $\alpha \mathrm{B}$-crystallin is upregulated by itself, as with exposures to hypertonic stress and tumor necrosis factor- $\alpha .^{58,62}$

That $\alpha \mathrm{B}$-crystallin might offer therapeutic benefit in $\mathrm{AxD}$ is suggested by two sets of observations. First, $\alpha \mathrm{B}$-crystallin promotes disassembly of preformed GFAP filaments in cell-free systems, ${ }^{63}$ and co-transfection of

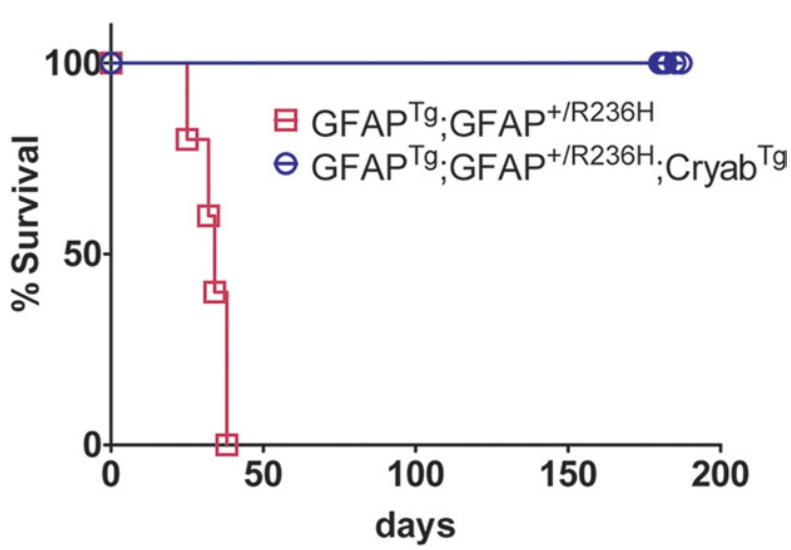

FIG. 3. Increased Cryab expression rescues lethal seizures, and reduces glial fibrillary acidic protein (GFAP) levels and Rosenthal fibers in GFAP ${ }^{\text {Tg; }}$ GFAP ${ }^{+/ R 236 H}$ mice. $100 \%$ of GFAP $^{\mathrm{Tg}} ; \mathrm{GFAP}^{+/ R 236 \mathrm{H}}$ mice die at an early age (for littermates shown [n = 5], median age of death = 34) compared with $100 \%$ survival in GFAP $^{\text {Tg;GFAP }}{ }^{+/ R 236 H}$ mice expressing Cryab $^{\text {Tg }}(\mathrm{n}=8 ; p=0.0002$, log rank Mantel-Cox test). (Reprinted with permission from Figure $3 A$ of Reference 45, Oxford University Press.)

$\alpha \mathrm{B}$-crystallin, along with wild type GFAP into cultured cells promotes dissolution of the filamentous aggregates that typically form in these situations. ${ }^{64,65}$ Using transgenic methods, increasing expression of $\alpha \mathrm{B}$-crystallin in the heart conferred protection against ischemic injury in vivo. ${ }^{66}$

Most recently, Hagemann et al. ${ }^{55}$ reported two key findings relating to the role of $\alpha \mathrm{B}$-crystallin in AxD. First, mice forming Rosenthal fibers from overexpression of wild-type human GFAP had increased mortality when crossed into an $\alpha \mathrm{B}$-crystallin-null background. This result supports the idea the AxD could result, in part, from depletion of $\alpha \mathrm{B}$-crystallin levels in astrocytes due to sequestration of this stress protein in Rosenthal fibers. Second, and most dramatically, forcing constitutive overexpression of $\alpha \mathrm{B}$-crystallin in astrocytes beyond its natural levels afforded complete rescue from the otherwise $100 \%$ mortality observed in a cross between two AxD mouse models (FIG. 3). The mechanisms by which $\alpha \mathrm{B}$-crystallin achieves this rescue are not clear, although recent data suggest that its binding to oligomeric forms of GFAP shifts the equilibrium to smaller forms and mitigates the effects of mutant GFAP on the proteasome. ${ }^{67}$ Whether pharmacologic enhancers of $\alpha \mathrm{B}$-crystallin expression can be found to mimic these transgenic studies is an area of active investigation.

\section{Enhancing protective stress responses-Nrf2}

A second stress pathway worth highlighting is that represented by the transcription factor Nrf2 (otherwise known as Nfe212). Normally Nrf2 is inactive and confined to the cytoplasm through binding to a Kelch-like protein, Keap-1. In response to a wide variety of stresses and especially oxidative stress, Nrf2 dissociates from 
Keap-1 and translocates to the nucleus, where it binds to a short anti-oxidant response element found in the promoters of a number of stress response genes, such as NQO1, glutathione-S-transferase, heme oxygenase-1, and ferritin heavy and light chains, thus activating their expression. ${ }^{68}$

In the CNS, activation of Nrf2 in response to toxic stress occurs primarily in astrocytes. ${ }^{69,70}$ Evidence that enhancing astrocytic expression of Nrf2 pathways in vivo might be neuroprotective has come from transplantation studies of adenoviral infected astrocytes into brains of animals given neurotoxic doses of malonate and 3-nitropropionic acid. ${ }^{71,72}$ Most recently, transgenic studies have demonstrated that constitutive enhancement of Nrf2 expression in astrocytes using the human GFAP promoter provides significant neuroprotection in two mouse models of neurodegenerative disease, the SOD1 G93A mutant model of amyotrophic lateral sclerosis, ${ }^{73}$ and the 1-methyl-4-phenyl-1,2,3,6-tetrahydropyridine toxin model of Parkinson's disease ${ }^{74}$ (see also reference 65 , in this volume).

We have found marked induction of the anti-oxidant response element pathways in human brain samples from Alexander diseased patients, as well as in our mouse models of AxD. ${ }^{19,32,55}$ It is interesting that one mechanism by which Nrf2 might be elevated is through impairment of the ubiquitin-proteasome system, ${ }^{75}$ a common feature of protein aggregation disorders that is likely occurring in AxD as well. ${ }^{21}$ Given the common activation of Nrf2 in a number of CNS disorders, the potential neuroprotective effects of enhancing expression, and the existence of several natural as well as synthetic inducers of Nrf2 activity that could have therapeutic applications, ${ }^{76}$ there is considerable appeal to further exploring its role in AxD.

\section{Minimizing detrimental downstream effects}

An alternative approach for treatment is to focus on the downstream detrimental effects of mutant GFAP expression, assuming one can identify those critical astrocyte function(s) that are compromised. As with many neurological disorders where astrocyte dysfunction is implicated, attention has focused on glutamate transport, since impaired transport could cause prolonged elevations of glutamate in the extracellular space with excitotoxic injury to neighboring cells (as previously noted). Glutamate transport in astrocytes is mediated predominately by EAAT2 (also known as Glt-1), and to a lesser extent by EAAT1 (also known as GLAST), with some variation depending on anatomical location and stage of development. Complete deficiency of EAAT2 in the mouse results in $94 \%$ reduction in glutamate transport in cerebral cortex, and $\sim 50 \%$ of the mice die from seizures by 6 weeks after birth. ${ }^{77}$ Interestingly, modest reductions in EAAT2 mRNA and protein are seen in the hippocampus of knock-in mouse models that carry a point mutation mimicking the $\mathrm{R} 239 \mathrm{H}$ mutation in human patients ( $236 \mathrm{H}$ in the mouse). ${ }^{55}$ These mice display enhanced severity and duration of seizures after exposure to kainic acid. Further reductions in EAAT2 expression are seen in mice that express both the point mutant and excess wild type human GFAP (from a transgene) that consistently die at 4 to 5 weeks after birth.

Reduced EAAT2 is also observed in humans with $\mathrm{AxD}$, where loss of hippocampal neurons correlate with reactive astrocytes and diminished transporter expression. $^{28}$ Astrocytic cell lines expressing R239C mutant GFAP show a significant decrease in EAAT2 expression with a reduced capacity to transport glutamate, and fail to rescue hippocampal neurons from glutamate toxicity in co-culture assays. ${ }^{28}$ One possible explanation for decreased EAAT2 expression is altered activation of NF$\kappa \mathrm{B}$, perhaps by reduction in signaling via phosphorylated Akt. ${ }^{41,78}$ EAAT2 is also regulated by ubiquitination and lysosomal degradation. ${ }^{79,80}$ Given the roles of proteasome function and autophagy in $\mathrm{AxD},{ }^{21,41}$ these pathways may also affect EAAT2 protein levels, in addition to reduced transcription.

Fortunately, EAAT2 previously emerged as a key therapeutic target for amyotrophic lateral sclerosis, and Rothstein et al. ${ }^{81}$ conducted a screen of drugs and compounds (approved by the Food and Drug Administration) using explant cultures of rat spinal cord to search for enhancers of EAAT2 expression. Several compounds belonging to the class of $\beta$-lactam antibiotics were effective in this screen. Further validation was performed using mice carrying an EGFP reporter under the control of the EAAT2 promoter. One of these antibiotics, ceftriaxone, increased expression of the EGFP reporter in the hippocampus of mice, and partially restored the decrease in EAAT2 levels that is otherwise seen in the spinal cords of the G93A SOD mouse mutant. Ceftriaxone is now in phase I clinical trials for amyotrophic lateral sclerosis to determine safety in the chronic dosing schedules that would be required in such a disease (as would also be true for $\mathrm{AxD}$ ). It is interesting, but puzzling, that several studies have now appeared showing the efficacy of ceftriaxone for the treatment of other disorders, sometimes without any change in expression of EAAT2 ${ }^{82}$ Hence, the beneficial effects of this antibiotic may involve pathways that have not yet been identified, although one possibility is activation of the Nrf2 pathway previously described. ${ }^{83}$

\section{HOW TO MEASURE SUCCESS?}

What criteria should we use to choose among these therapeutic strategies? Cell cultures have provided most of the preliminary data in support of these approaches to 
therapy, but these have primarily involved the use of transfected cell lines, which are convenient but highly artificial in several respects. Even primary cultures of astrocytes derived from one of the existing mouse models of $\mathrm{AxD}$, while producing aggregates that display the same ultrastructural characteristics of bona fide Rosenthal fibers, ${ }^{84}$ still consist of cells that are isolated from their normal anatomical context and interactions with other cell types. Mouse models have been created via both transgenic and knock-in approaches that reproduce key aspects of the Alexander phenotype, particularly the formation of Rosenthal fibers identical to those found in human disease ${ }^{18}$ and increased seizure susceptibility. ${ }^{19,20}$ However, none of the existing mouse models developed a leukodystrophy. Nevertheless, despite the imperfections of the mouse models, results of testing in vivo will have more validity than findings developed solely from cultured astrocytes.

Should any proposed therapy reach the stage of clinical trials, a number of other issues arise. The rarity of the disorder makes the idea of double-blind, placebocontrolled trials unrealistic. Instead, patients will likely have to serve as their own controls by comparing status pre- and post-treatment. However, although existing MRI criteria are highly reliable as diagnostic tools, they are not suitable for the purposes of quantifying disease severity or monitoring disease progression. Hence, any future clinical trials would be greatly facilitated by the identification of biomarkers that could serve as surrogate indicators of the response to therapy. One potential biomarker is GFAP itself. GFAP is normally present only at low levels in the CSF, but increases in the context of a number of diseases or injuries. ${ }^{85}$ Kyllerman et al. ${ }^{86}$ studied three AxD patients, and found elevations of GFAP in all, although only one measurement was made for each patient. Studies are now underway to replicate these studies in a larger cohort of patients and to determine whether GFAP is elevated in more convenient body fluids, such as blood.

\section{CONCLUSIONS}

The past 10 years have witnessed enormous progress in the understanding of $\mathrm{AxD}$, with clinical practice transformed by the relative ease of genetic diagnosis and new ideas coming forth in regard to the effects that expression of mutant GFAP have on astrocyte biology. Whether these ideas can be translated into an effective therapy is the next, and perhaps most difficult, challenge.

Acknowledgements: We thank Michael Brenner, ShingYan Chiu, and James Goldman for comments on the article. This work was supported by the National Institutes of Health (Grants No. HD046599, No. NS060120, and No. NS042803 to
AM, and Grant No. HD03352 to the Waisman Center). Additional support was provided by the Palamaro and Juanma Funds.

\section{REFERENCES}

1. Brenner M, Goldman JE, Quinlan RA, Messing A. Alexander disease: a genetic disorder of astrocytes. In: Astrocytes in (Patho) Physiology of the Nervous System, Parpura V \& Haydon PG, eds. Springer, New York, 2009:591-648.

2. Alexander WS. Progressive fibrinoid degeneration of fibrillary astrocytes associated with mental retardation in a hydrocephalic infant. Brain 1949;72:373-81.

3. Herndon RM, Rubinstein LJ, Freeman JM, Mathieson G. Light and electron microscopic observations on Rosenthal fibers in Alexander's disease and in multiple sclerosis. J Neuropathol Exp Neurol 1970;29:524-51.

4. Iwaki T, Kume-Iwaki A, Liem RKH, Goldman JE. $\alpha$ B-Crystallin is expressed in non-lenticular tissues and accumulates in Alexander's disease brain. Cell 1989;57:71-8.

5. Head MW, Goldman JE. Small heat shock proteins, the cytoskeleton, and inclusion body formation. Neuropathol Appl Neurobiol 2000;26:304-12.

6. Brenner $\mathrm{M}$, Johnson $\mathrm{AB}$, Boespflug-Tanguy $\mathrm{O}$, Rodriguez $\mathrm{D}$, Goldman JE, Messing A. Mutations in GFAP, encoding glial fibrillary acidic protein, are associated with Alexander disease. Nature Genet 2001;27:117-20.

7. Quinlan RA, Brenner M, Goldman J, Messing A. GFAP and its role in Alexander disease. Exp Cell Res 2007;313:2077-87.

8. Perng MD, Su M, Wen SF, et al. The Alexander disease-causing GFAP mutant, R416W, accumulates into Rosenthal fibers by a pathway involving filament aggregation and the association of $\alpha$ B-crystallin and HSP27. Am J Hum Genet 2006;79:197-213.

9. van der Knaap MS, Ramesh V, Schiffmann R, et al. Alexander disease: ventricular garlands and abnormalities of the medulla and spinal cord. Neurology 2006;66:494-8.

10. Barkovich AJ, Messing A. Alexander disease: not just a leukodystrophy anymore. Neurology 2006;66:468-9.

11. van der Knaap MS, Naidu S, Breiter SN, et al. Alexander disease: diagnosis with MR imaging. Am J Neuroradiol 2001;22:541-52.

12. van der Knaap MS, Salomons GS, Li R, et al. Unusual variants of Alexander disease. Ann Neurol 2005;57:327-38.

13. Staba MJ, Goldman S, Johnson FL, Huttenlocher PR. Allogeneic bone marrow transplantation for Alexanders disease. Bone Marrow Transplant 1997;20:247-9.

14. Ishigaki K, Ito Y, Sawaishi Y, et al. TRH therapy in a patient with juvenile Alexander disease. Brain Dev 2006;28:663-7.

15. Sechi G, Matta M, Deiana GA, et al. Ceftriaxone has a therapeutic role in Alexander disease. Prog Neuropsychopharmacol Biol Psychiatry 2010;34:416-7.

16. Quinlan R, Hutchison C, Lane B. Intermediate filament proteins. Protein Profile 1995;2:801-952.

17. Mignot C, Boespflug-Tanguy O, Gelot A, Dautigny A, Pham-Dinh $\mathrm{D}$, Rodriguez D. Alexander disease: putative mechanisms of an astrocytic encephalopathy. Cell Mol Life Sci 2004;61:369-85.

18. Messing A, Head MW, Galles K, Galbreath EJ, Goldman JE, Brenner M. Fatal encephalopathy with astrocyte inclusions in GFAP transgenic mice. Am J Pathol 1998;152:391-8.

19. Hagemann TL, Connor JX, Messing A. Alexander disease-associated glial fibrillary acidic protein mutations in mice induce Rosenthal fiber formation and a white matter stress response. J Neurosci 2006;26:11162-73.

20. Tanaka KF, Takebayashi H, Yamazaki Y, et al. The murine model of Alexander disease: analysis of GFAP aggregate formation and its pathological significance. Glia 2007;55:617-31.

21. Tang G, Xu Z, Goldman JE. Synergistic effects of the SAPK/JNK and the proteasome pathway on glial fibrillary acidic protein (GFAP) accumulation in Alexander disease. J Biol Chem 2006; 281:38634-43.

22. Tian RJ, Gregor M, Wiche G, Goldman JE. Plectin regulates the organization of glial fibrillary acidic protein in Alexander disease. Am J Pathol 2006;168:888-97. 
23. Zatloukal K, Stumptner C, Fuchsbichler A, et al. p62 is a common component of cytoplasmic inclusions in protein aggregation diseases. Am J Pathol 2002;160:255-63.

24. Fern R, Möller T. Rapid ischemic cell death in immature oligodendrocytes: a fatal glutamate release feedback loop. J Neurosci 2000;20:34-42.

25. Follett PL, Rosenberg PA, Volpe JJ, Jensen FE. NBQX attenuates excitotoxic injury in developing white matter. J Neurosci 2000;20: 9235-41.

26. Li S, Stys PK. Mechanisms of ionotropic glutamate receptormediated excitotoxicity in isolated spinal cord white matter. J Neurosci 2000;20:1190-8.

27. Micu I, Jiang Q, Coderre E, et al. NMDA receptors mediate calcium accumulation in myelin during chemical ischaemia. Nature 2006;439:988-92.

28. Tian R, Wu X, Hagemann TL, et al. Alexander disease mutant GFAP compromises glutamate transport in astrocytes. J Neuropathol Exp Neurol 2010;69:335-45.

29. Chiu SY, Kriegler S. Neurotransmitter-mediated signaling between axons and glial cells. Glia 1994;11:191-200.

30. Li S, Mealing GA, Morley P, Stys PK. Novel injury mechanism in anoxia and trauma of spinal cord white matter: glutamate release via reverse $\mathrm{Na}+$-dependent glutamate transport. J Neurosci 1999; 19:RC16.

31. Butt AM, Jenkins HG. Morphological changes in oligodendrocytes in the intact mouse optic nerve following intravitreal injection of tumour necrosis factor. J Neuroimmunol 1994;51:27-33.

32. Hagemann TL, Gaeta SA, Smith MA, Johnson DA, Johnson JA, Messing A. Gene expression analysis in mice with elevated glial fibrillary acidic protein and Rosenthal fibers reveals a stress response followed by glial activation and neuronal dysfunction. Hum Mol Genet 2005;14:2443-58.

33. Lutz SE, Zhao Y, Gulinello M, Lee SC, Raine CS, Brosnan CF. Deletion of astrocyte connexins 43 and 30 leads to a dysmyelinating phenotype and hippocampal CA1 vacuolation. J Neurosci 2009;29:7743-52.

34. Gomi H, Yokoyama T, Fujimoto K, et al. Mice devoid of the glial fibrillary acidic protein develop normally and are susceptible to scrapie prions. Neuron 1995; 14:29-41.

35. Pekny M, Levéen P, Pekna M, et al. Mice lacking glial fibrillary acidic protein display astrocytes devoid of intermediate filaments but develop and reproduce normally. EMBO J 1995;14:1590-8.

36. McCall MA, Gregg RG, Behringer RR, et al. Targeted deletion in astrocyte intermediate filament (Gfap) alters neuronal physiology. Proc Natl Acad Sci USA 1996;93:6361-6.

37. Liedtke W, Edelmann W, Chiu F-C, Kucherlapati R, Raine CS. Experimental autoimmune encephalomyelitis in mice lacking glial fibrillary acidic protein is characterized by a more severe clinical course and an infiltrative central nervous system lesion. Am J Pathol 1998;152:251-9.

38. Kadurugamuwa JL, Modi K, Coquoz O, et al. Reduction of astrogliosis by early treatment of pneumococcal meningitis measured by simultaneous imaging, in vivo, of the pathogen and host response. Infect Immun 2005;73:7836-43.

39. Cho W, Hagemann TL, Johnson DA, Johnson JA, Messing A. Dual transgenic reporter mice as a tool for monitoring expression of GFAP. J Neurochem 2009;110:343-51.

39a. Cho W, Brenner M, Peters N, Messing A. Drug screening to identify suppressors of GFAP expression. Hum Mol Genet 2010; 19:3169-78

40. Huang L, Chen CH. Proteasome regulators: activators and inhibitors. Curr Med Chem 2009;16:931-9.

41. Tang G, Yue Z, Talloczy Z, et al. Autophagy induced by Alexander disease-mutant GFAP accumulation is regulated by $\mathrm{p} 38 /$ MAPK and mTOR signaling pathways. Hum Mol Genet 2008;17: 1540-55.

42. Ravikumar B, Vacher C, Berger Z, et al. Inhibition of mTOR induces autophagy and reduces toxicity of polyglutamine expansions in fly and mouse models of Huntington disease. Nature Genet 2004;36:585-95.

43. Meikle L, Pollizzi K, Egnor A, et al. Response of a neuronal model of tuberous sclerosis to mammalian target of rapamycin (mTOR) inhibitors: Effects on mTORC1 and Akt signaling lead to improved survival and function. J Neurosci 2008;28:5422-32.

44. Zeng LH, Xu L, Gutmann DH, Wong M. Rapamycin prevents epilepsy in a mouse model of tuberous sclerosis complex. Ann Neurol 2008;63:444-53.

45. Menzies FM, Huebener J, Renna M, Bonin M, Riess O, Rubinsztein DC. Autophagy induction reduces mutant ataxin-3 levels and toxicity in a mouse model of spinocerebellar ataxia type 3 . Brain 2010;133:93-104.

46. Harrison DE, Strong R, Sharp ZD, et al. Rapamycin fed late in life extends lifespan in genetically heterogeneous mice. Nature 2009; 460:392-5.

47. Carmichael J, Sugars KL, Bao YP, Rubinsztein DC. Glycogen synthase kinase-3 beta inhibitors prevent cellular polyglutamine toxicity caused by the Huntington's disease mutation. J Biol Chem 2002;277:33791-8.

48. Sarkar S, Floto RA, Berger Z, et al. Lithium induces autophagy by inhibiting inositol monophosphatase. J Cell Biol 2005;17:1101-11.

49. Fornai F, Longone P, Cafaro L, et al. Lithium delays progression of amyotrophic lateral sclerosis. Proc Natl Acad Sci USA 2008; 105:2052-7.

50. Berger Z, Ttofi EK, Michel CH, et al. Lithium rescues toxicity of aggregate-prone proteins in Drosophila by perturbing Wnt pathway. Hum Mol Genet 2005;14:3003-11.

51. Noble W, Planel E, Zehr C, et al. Inhibition of glycogen synthase kinase-3 by lithium correlates with reduced tauopathy and degeneration in vivo. Proc Natl Acad Sci USA 2005;102:6990-5.

52. Sarkar S, Krishna G, Imarisio S, Saiki S, O'Kane CJ, Rubinsztein DC. A rational mechanism for combination treatment of Huntington's disease using lithium and rapamycin. Hum Mol Genet 2008; $17: 170-8$.

53. Iwaki T, Iwaki A, Tateishi J, Sakaki Y, Goldman JE. Alpha Bcrystallin and $27-\mathrm{kd}$ heat shock protein are regulated by stress conditions in the central nervous system and accumulate in Rosenthal fibers. Am J Pathol 1993;143:487-95.

54. Iwaki T, Wisniewski T, Iwaki A, et al. Accumulation of $\alpha \mathrm{B}$ crystallin in central nervous system glia and neurons in pathologic conditions. Am J Pathol 1992;140:345-56.

55. Hagemann TL, Boelens W, Wawrousek E, Messing A. Suppression of GFAP toxicity by $\alpha \mathrm{B}$-crystallin in mouse models of Alexander disease. Hum Mol Genet 2009;18:1190-9.

56. Iwaki T, Iwaki A, Tateishi J, Goldman JE. Sense and antisense modification of glial $\alpha \mathrm{B}$-crystallin production results in alterations of stress fiber formation and thermoresistance. J Cell Biol 1994; 125:1385-93.

57. Michl M, Ouyang N, Fraek ML, Beck FX, Neuhofer W. Expression and regulation of $\alpha \mathrm{B}$-crystallin in the kidney in vivo and in vitro. Pflugers Arch Eur J Physiol 2006;452:387-95.

58. Head MW, Hurwitz L, Goldman JE. Transcriptional regulation of $\alpha \mathrm{B}$-crystallin in astrocytes: analysis of HSF and AP1 activation by different types of physiological stress. J Cell Sci 1996;109:1029-39.

59. Kegel KB, Iwaki A, Iwaki T, Goldman JE. $\alpha$ B-crystallin protects glial cells from hypertonic stress. Am J Physiol Cell Physiol 1996; 270:C903-9.

60. Morrison LE, Hoover HE, Thuerauf DJ, Glembotski CC. Mimicking phosphorylation of $\alpha \mathrm{B}$-crystallin on serine-59 is necessary and sufficient to provide maximal protection of cardiac myocytes from apoptosis. Circ Res 2003;92:203-11.

61. Martin JL, Mestril R, Hilal-Dandan R, Brunton LL, Dillmann WH. Small heat shock proteins and protection against ischemic injury in cardiac myocytes. Circulation 1997;96:4343-8.

62. Head MW, Corbin E, Goldman JE. Coordinate and independent regulation of alpha B-crystallin and hsp27 expression in response to physiological stress. J Cell Physiol 1994;159:41-50.

63. Nicholl ID, Quinlan RA. Chaperone activity of $\alpha$-crystallins modulates intermediate filament assembly. EMBO J 1994;13:945-53.

64. Koyama Y, Goldman JE. Formation of GFAP cytoplasmic inclusions in astrocytes and their disaggregation by $\alpha \mathrm{B}$-crystallin. Am J Pathol 1999;154:1563-72.

65. Bachetti T, Caroli F, Bocca P, et al. Mild functional effects of a novel GFAP mutant allele identified in a familial case of adultonset Alexander disease. Eur J Hum Genet 2008;16:462-70. 
66. Ray PS, Martin JL, Swanson EA, Otani H, Dillmann WH, Das DK. Transgene overexpression of $\alpha \mathrm{B}$ crystallin confers simultaneous protection against cardiomyocyte apoptosis and necrosis during myocardial ischemia and reperfusion. FASEB J 2001;15:393-402.

67. Tang G, Perng MD, Wilk S, Quinlan R, Goldman JE. Oligomers of mutant glial fibrillary acidic protein (GFAP) inhibit the proteasome system in Alexander disease astrocytes, and the small heat shock protein, $\alpha \mathrm{B}$-crystallin, reverses the inhibition. J Biol Chem 2010; 285:10527-37.

68. Nguyen T, Yang CS, Pickett CB. The pathways and molecular mechanisms regulating Nrf2 activation in response to chemical stress. Free Radic Biol Med 2004;37:433-41.

69. Johnson DA, Andrews GK, Xu W, Johnson JA. Activation of the antioxidant responsive element in primary cortical neuronal cultures derived from transgenic reporter mice. J Neurochem 2002; 81:1233-41.

70. Kraft AD, Johnson DA, Johnson JA. Nuclear factor E2-related factor 2-dependent antioxidant response element activation by tertbutylhydroquinone and sulforaphane occurring preferentially in astrocytes conditions neurons against oxidative insult. J Neurosci 2004;24:1101-12.

71. Calkins MJ, Jakel RJ, Johnson DA, Chan KM, Kan YW, Johnson JA. Protection from mitochondrial complex II inhibition in vitro and in vivo by Nrf2-mediated transcription. Proc Natl Acad Sci USA 2005;102:244-9.

72. Shih AY, Imbeault S, Barakauskas V, et al. Induction of the Nrf2-driven antioxidant response confers neuroprotection during mitochondrial stress in vivo. J Biol Chem 2005;280:22925-36.

73. Vargas MR, Johnson DA, Sirkis DW, Messing A, Johnson JA. Nrf2 activation in astrocytes protects against neurodegeneration in mouse models of familial amyotrophic lateral sclerosis. J Neurosci 2008;25:13574-81.

74. Chen PC, Vargas MR, Pani AK, et al. Nrf2-mediated neuroprotection in the MPTP mouse model of Parkinson's disease: critical role for the astrocyte. Proc Natl Acad Sci USA 2009;106:2933-8.

75. Stewart D, Killeen E, Naquin R, Alam S, Alam J. Degradation of transcription factor Nrf2 via the ubiquitin-proteasome pathway and stabilization by cadmium. J Biol Chem 2003;278:2396-402.
76. JW, Fahey Zhang Y, Talalay P. Broccoli sprouts: an exceptionally rich source of inducers of enzymes that protect against chemical carcinogens. Proc Natl Acad Sci USA 1997;94:10367-72.

77. Tanaka K, Watase K, Manabe T, et al. Epilepsy and exacerbation of brain injury in mice lacking the glutamate transporter GLT-1. Science 1997;276:1699-702.

78. Li LB, Toan SV, Zelenaia O, et al. Regulation of astrocytic glutamate transporter expression by Akt: evidence for a selective transcriptional effect on the GLT-1/EAAT2 subtype. J Neurochem 2006;97:759-71.

79. Sheldon AL, González MI, Krizman-Genda EN, Susarla BT, Robinson MB. Ubiquitination-mediated internalization and degradation of the astroglial glutamate transporter, GLT-1. Neurochem Int 2008;53:296-308.

80. Susarla BT, Robinson MB. Internalization and degradation of the glutamate transporter GLT-1 in response to phorbol ester. Neurochem Int 2008;52:709-22.

81. Rothstein JD, Patel S, Regan MR, et al. $\beta$-Lactam antibiotics offer neuroprotection by increasing glutamate transporter expression. Nature 2005;433:73-7.

82. Melzer N, Meuth SG, Torres-Salazar D, et al. A $\beta$-lactam antibiotic dampens excitotoxic inflammatory CNS damage in a mouse model of multiple sclerosis. PLoS ONE 2008;3:e3149.

83. Lewerenz J, Albrecht P, Tien MLT, et al. Induction of Nrf2 and $\mathrm{xCT}$ are involved in the action of the neuroprotective antibiotic ceftriaxone in vitro. J Neurochem 2009;111:332-43.

84. W, Cho Messing A. Properties of astrocytes cultured from GFAP overexpressing and GFAP mutant mice. Exp Cell Res 2009;315:1260-72.

85. Liem RKH, Messing A. Dysfunctions of neuronal and glial intermediate filaments in disease. J Clin Invest 2009;119:1814 24.

86. Kyllerman M, Rosengren L, Wiklund L-M, Holmberg E. Increased levels of GFAP in the cerebrospinal fluid in three subtypes of genetically confirmed Alexander disease. Neuropediatrics 2005; 36:319-23. 